\title{
Charge Injection Devices
}

\author{
Jaakko Härkönen ${ }^{1}$ on behalf of RD39 Collaboration \\ Helsinki Institute of Physics \\ Gustaf Hällströmin katu 2, 00014 Helsinki, Finland \\ E-mail: jaakko.haerkoenen@cern.ch
}

\begin{abstract}
The concept of Charge Injected Detectors (CID) has been developed and investigated by the CERN RD39 Collaboration for a considerable period of time. The CID is based on a balance between the trapping and detrapping processes in heavily irradiated semiconductor detector material. Radiation-induced defects trap charge carriers. The counter process of trapping, detrapping, is exponentially dependent on temperature. Thus, if the temperature is lowered, the trapped charge may occupy the radiation defect for considerably long time. This modifies the electric field distribution in an irradiated semiconductor. The trapped charge results in a continuous electric field through the entire thickness of a detector. The experimental results from RD39 show that a standard silicon strip detector, irradiated to $1 \times 10^{15} \mathrm{n}_{\mathrm{eq}} / \mathrm{cm}^{2}$ fluence, operated under CID mode has a relative charge collection efficiency (CCE) more than 33\% while having a signal-to-noise (SNR) being slightly less than than 10 . The CID operation of such a sensor requires a temperature of about $-50^{\circ} \mathrm{C}$. The amount of injected forward current is comparable with the leakage current of a similarly irradiated detector operated with reverse bias voltage at about $-20^{\circ} \mathrm{C}$.
\end{abstract}

The 20th Anniversary International Workshop on Vertex Detectors - Vertex 2011 Rust, Lake Neusiedl, Austria (June 19-24, 2011)

\footnotetext{
${ }^{1}$ Speaker
} 


\section{Introduction}

Radiation hardness over $1 \times 10^{16} \mathrm{n}_{\mathrm{eq}} / \mathrm{cm}^{2}$ ( $\mathrm{n}_{\mathrm{eq}}$ refers to $1 \mathrm{MeV}$ neutron equivalent fluence) will be required in the future HEP experiments. The most important measure of the detector's radiation hardness is the Charge Collection Efficiency (CCE), which is affected by the sensitive volume of the detector (depletion depth) and the charge trapping into the radiation-induced trapping centers. At this fluence the needed full depletion voltage $\left(\mathrm{V}_{\mathrm{fd}}\right)$ would be in the scale of thousands of volts for a $300 \mu \mathrm{m}$ thick $\mathrm{Si}$ detector operated at or near room temperature. Moreover, the charge carrier trapping will limit the charge collection depth to an effective range of $20 \mu \mathrm{m}$ to $30 \mu \mathrm{m}$ regardless of the depletion depth. In order to maintain an acceptable CCE in the Super-LHC radiation environment, one has to solve both problems simultaneously.

The approach of the CERN RD39 collaboration is to inject electrons through the $\mathrm{n}^{+}$ contact in a Si detector by forward biasing the $\mathrm{p}^{+} / \mathrm{n} / \mathrm{n}^{+}$detector that has been irradiated beyond the space-charge-sign-inversion (SCSI), where the main junction is located near the $\mathrm{n}^{+}$contact. By injecting electrons with a substantial current, one can reach a dynamic equilibrium between trapping and detrapping at a given temperature, in which most of electron traps are filled up, and therefore no longer active in trapping free electrons generated by particles to be detected. Under these conditions, the electric field extends through the entire detector thickness regardless of the applied voltage or concentration of deep levels. Depending on the point of injection, the electric field increases towards the back plane of the detector or vice versa. The amplitude of the electric field is proportional to the square root of the distance from the injecting junction [1,2].

Low temperature $\left(-50^{\circ} \mathrm{C}\right.$ for example) and a high concentration of deep levels are required in order to establish the stable electric field favorable for detector operation by charge injection. The CID detector cannot operate at the room temperature as a particle detector. In practice, CID would require a S-LHC irradiation fluence, i.e. $>1 \times 10^{15} \mathrm{n}_{\mathrm{eq}} / \mathrm{cm}^{2}$ for its operation.

\section{Current in a CID detector}

It is obvious that tracker systems operating in harsh radiation environments require cooling in order to remove the excess heat induced by the readout electronics and in order to suppress the shot noise caused by leakage current due to the radiation defects. This is the case regardless of whether sensors are intended to be operated under reverse bias or CID mode. If the charge injection is realized by forward biasing the pn-junction, the current needed is determined by a balance between the trapping and its counter process detrapping, i.e. emission of trapped charge carrier back into signal transportation $[3,4,5]$. The time constants of these competitive processes are given by 


$$
\begin{gathered}
\tau_{\text {trapping }}=\frac{1}{\sigma_{e, h} V_{t h} N_{t}} \\
\tau_{\text {detrapping }}=\frac{1}{\sigma_{e, h} V_{t h} e^{\frac{-E_{t}}{k T}}}
\end{gathered}
$$

where $\tau_{\text {trapping }}$ is the time constant for trapping, $\tau_{\text {detrapping }}$ is the time constant for detrapping, $\sigma_{\mathrm{e}, \mathrm{h}}$ is the capture cross section of the trap, $v_{t h}$ is the thermal velocity of charge carriers, $N_{T}$ is the concentration of traps, $N_{C}$ the electric state density in the conduction band, and $E_{t}$ the trap energy level in the band gap. It can be seen that the trapping does not depend on the temperature, while the detrapping contains an exponential temperature dependence. This means that the amount of forward current needed to give efficient charge injection decreased rapidly with decreasing temperature. On the other hand, under hash radiation environment the trapping is the limiting factor of charge collection. Thus, the charge transportation must be as fast as possible in order avoid particle generated charge being lost into radiation defects by trapping. This in turn requires relatively high bias voltage in order to maintain sufficient drift velocity through the thickness of detector's sensitive volume

$$
v_{\text {drift }}=\mu_{e, h} E(x)
$$

where $\mu_{e, h}$ is the mobility of the electrons and holes, respectively. The equations (1) and (2) imply that the CID cannot operate at room temperature as a particle detector, at this temperature a very a very high forward current would be required to fill the defects since the trapped charge would almost immediately be emitted back to current transportation by the detrapping process. As a result, a very small voltage would be required to inject this current and charge carriers would drift with a very small velocity. For the same reasons, an unirradiated silicon detector cannot operate as CID, not even at low temperature. In an unirradiated detector the concentration of deep levels is usually negligibly small. Thus, all the deep levels would be filled with a very small current injection injection, resulting in an insufficiently small electric field for efficienct charge drift. In practice, the CID would require a S-LHC irradiation fluence, i.e. $>1 \times 10^{15} \mathrm{n}_{\mathrm{eq}} / \mathrm{cm}^{2}$ for its operation. This is illustrated in figure 1., which shows simulated and measured IV characteristics of CID detectors. 


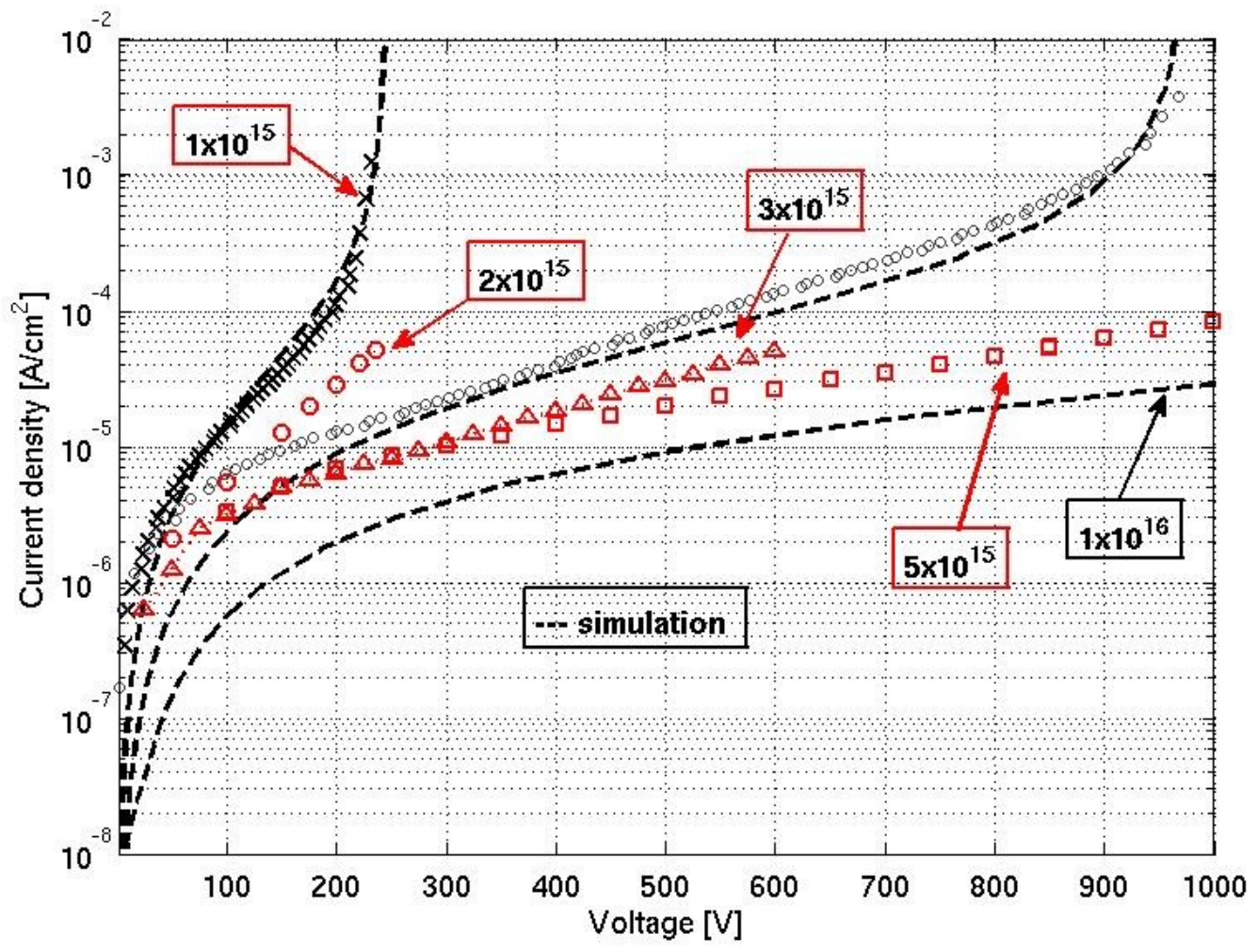

Figure 1. IV characteristics of CID detectors. The red open symbols ( $\Delta \Delta$ ) correspond to strip detectors with 768 channels. The black dashed lines (---) are for simulations of the current and the symbol $(X)$ stands for measurement of diodes. The measurements have been carried out at $-50^{\circ} \mathrm{C}$ temperature. The current values indicated for strip detectors are recorded from CAEN power supply biasing the detector module with APV25 readout [6].

\section{Signal from CID detector}

The RD39 Collaboration has constructed a Cryogenic Transient Current Technique (C-TCT) measurement setup, which is capable to operate below liquid nitrogen temperatures [7]. The CTCT is an effective research tool to study heavily irradiated silicon detectors. The TCT measurement is based on the detection of the dominant type of charge carrier, electron or hole, which drifts across the whole detector thickness after being excited by a photon. This is achieved by illuminating (in a $\mathrm{n}+/ \mathrm{p}-\mathrm{p}+$ device) the front ( $\mathrm{n}+$ implant) or back ( $\mathrm{p}+$ implant) side of the detector with a red laser, whose light creates electron-hole pairs close to the device surface. When the front-side of the detector is illuminated, the electrons move only a few microns (small signal) and are gathered to the $\mathrm{n}+$-electrode so fast that the signal is damped by the rise-time of the data acquisition electronics and therefore the measured signal is mainly coming from the holes that travel a longer distance through the silicon bulk. By analyzing the 
transient, it is possible to extract the full depletion voltage, effective trapping time, electric field distribution and the sign of the space charge in the silicon bulk [8,9]. Furthermore, the CCE can be determined if the detector is illuminated by sub band gap infrared light, which provides homogeneous generation of electron hole pairs through the entire bulk of silicon and therefore simulates minimum ionizing particles [10]. The CCE can be obtained by comparing the signal from irradiated detector with signal measured from non-irradiated sample. An example of such a measurement is shown in Figure 2.

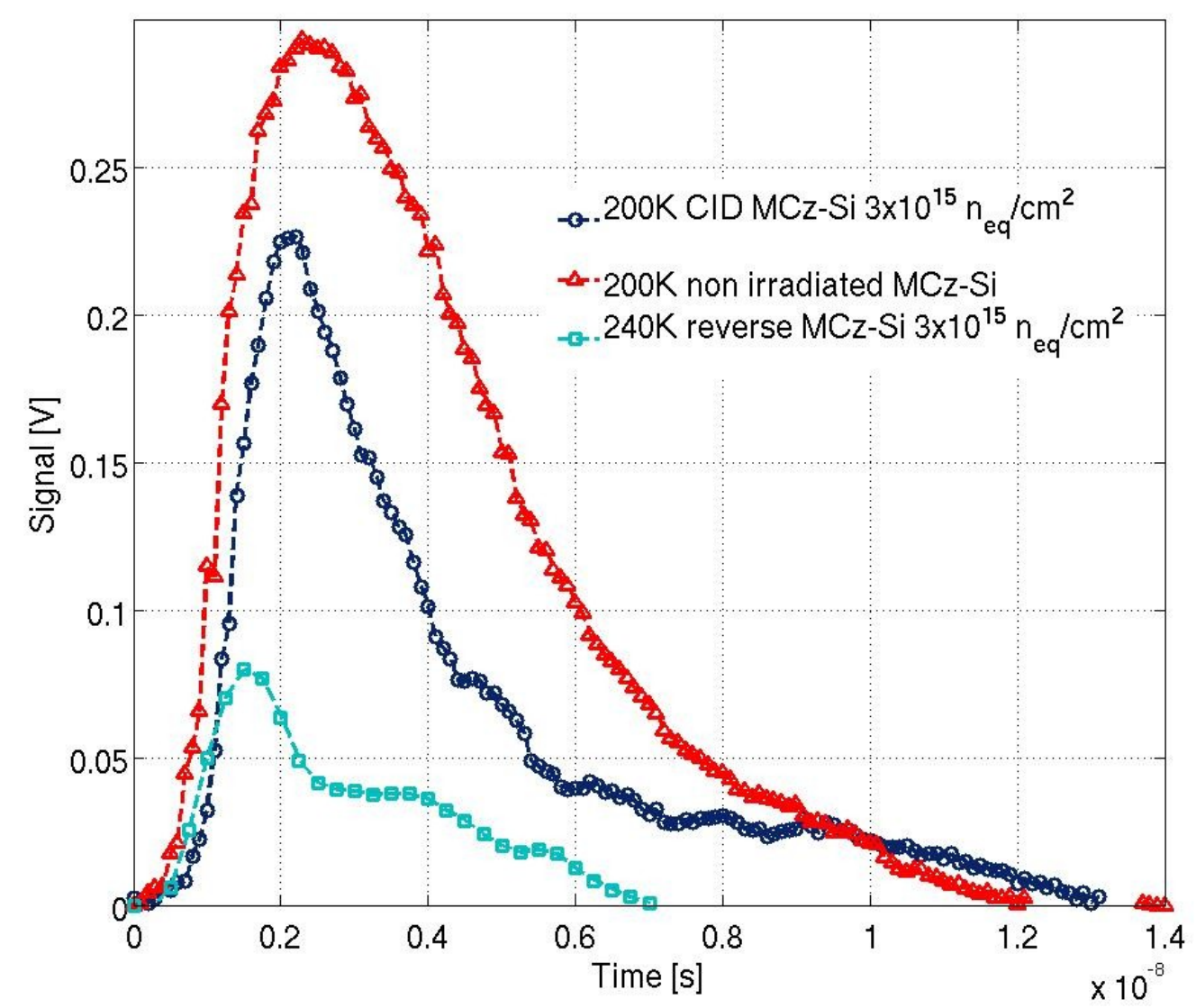

Figure 2. TCT signals exicited by an infrared (IR) laser of a non-irradiated (red triangle), CID detector with $500 \mathrm{~V}$ bias (open circle) and the same detector under $500 \mathrm{~V}$ reverse bias (open square). The irradiation fluence is $3 \times 10^{15} \mathrm{n}_{\mathrm{eq}} / \mathrm{cm}^{2}$.

\section{Test beam measurements for CID detectors}

The $\mathrm{n}$ and $\mathrm{p}$-type $\left(\mathrm{p}^{+}\right.$strip implant on $\mathrm{n}$-type wafer and $\mathrm{n}^{+}$strip implant on $\mathrm{p}$-type wafer) detectors were processed at the Micronova micro and nanotechnology center of Helsinki 
University of Technology. The starting material of the detectors was $100 \mathrm{~mm}$ diameter doubleside-polished $300 \pm 2 \mu \mathrm{m}$-thick $<100>\mathrm{n}$ and p-type Magnetic Czochralski silicon (MCz-Si) wafers. The nominal resistivity, measured by the four point probe method, of the wafers is $900-$ $1100 \Omega \mathrm{cm}$ for $\mathrm{n}$-type wafers and $3 \mathrm{k} \Omega \mathrm{cm}$ for $\mathrm{p}$-type wafers, respectively. The size of the strip detectors is $4 \mathrm{~cm} \times 4 \mathrm{~cm}$ and there are $76810 \mu \mathrm{m}$ wide strips with $50 \mu \mathrm{m}$ pitch [11].

The $\mathrm{p}$ and $\mathrm{n}$-type sensors were irradiated to the fluencies of $2 \times 10^{15} \mathrm{n}_{\mathrm{eq}} / \mathrm{cm}^{2}$ and $5 \times 10^{15} \mathrm{n}_{\mathrm{eq}} / \mathrm{cm}^{2}$, respectively, by $26 \mathrm{MeV}$ protons at Karslruhe. After the irradiations the detectors were wire bonded at the CERN bonding laboratory to the CMS APV25 hybrid [12] via pitch adapters. An overview of a CID module is shown in figure 3.

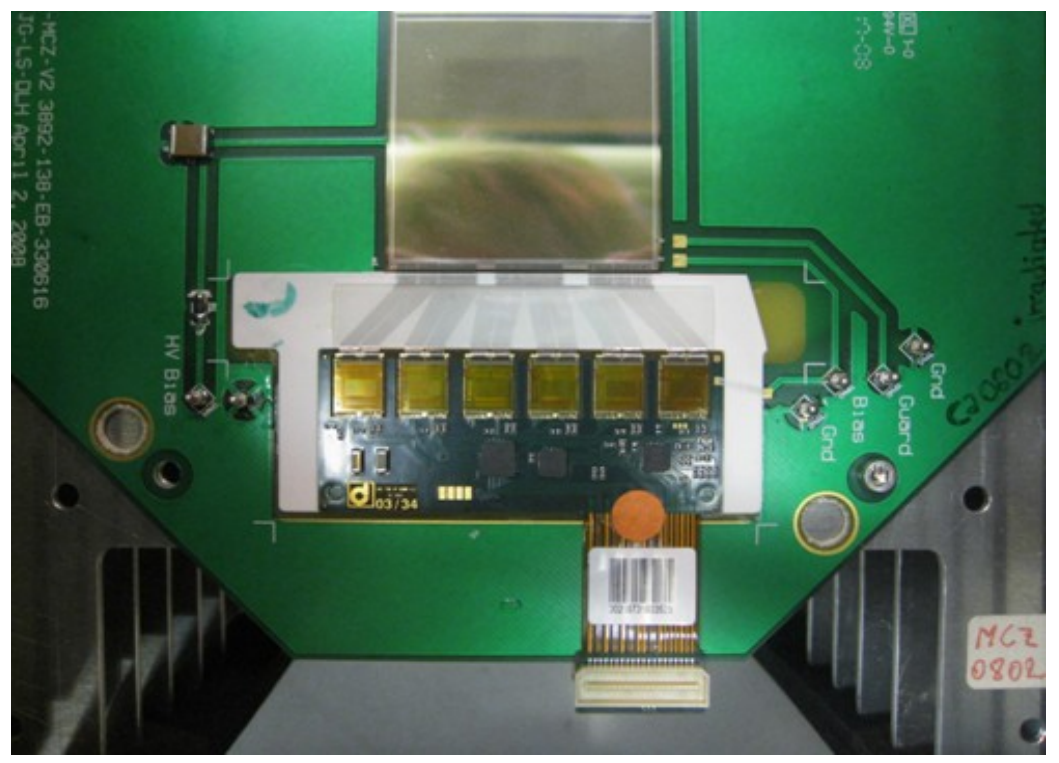

Figure 3.768 channels $4 \mathrm{~cm} \times 4 \mathrm{~cm}$ strip detector attached to the CMS readout module.

During the operation, the CID modules were placed on an external cold finger next to the SiBT telescope. The experimental arrangement is shown in our previous reports [13,14]. In the following plots, the temperature of cold finger of the separate cold box was $-53^{\circ} \mathrm{C}$ unless otherwise stated.

Figure 4. shows the collected charge as a function of bias voltage of $5 \times 10^{15}$ and $3 \times 10^{15} \mathrm{n}_{\mathrm{eq}} / \mathrm{cm}^{2}$ irradiated $\mathrm{p}^{+} / \mathrm{n}^{-} / \mathrm{n}^{+}$CID detectors at different temperatures . 


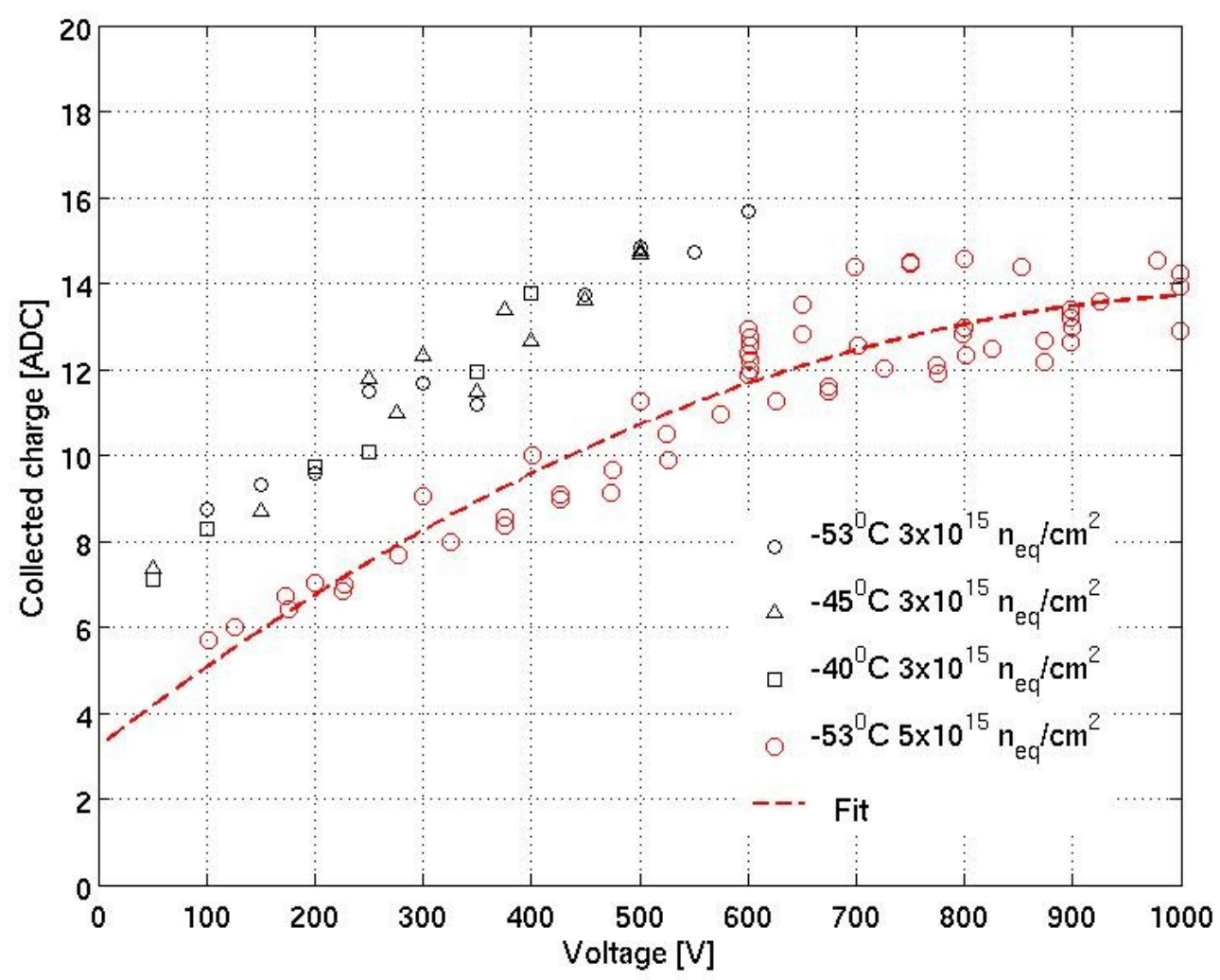

Figure 4. Collected charge of heavily irradiated CID detectors as a function of bias voltage. The red symbols are for $5 \times 10^{15} \mathrm{n}_{\mathrm{eq}} / \mathrm{cm}^{2}$ and black symbols for $5 \times 10^{15} \mathrm{n}_{\mathrm{eq}} / \mathrm{cm}^{2}$ irradiated detectors.

It can be seen in figure 4 that the collected charge is at $600 \mathrm{~V} 12.5 \mathrm{ADC}$ and $16 \mathrm{ADC}$ for $5 \times 10^{15}$ $\mathrm{n}_{\mathrm{eq}} / \mathrm{cm}^{2}$ (red symbols) and for $5 \times 10^{15} \mathrm{n}_{\mathrm{eq}} / \mathrm{cm}^{2}$ irradiated detectors (black symbols), respectively. The charge recorded from beam telescope's reference planes is in on average 40 ADC [15]. Thus, the relative CCE can be considered to be $30 \%$ and $40 \%$ for these CID devices.

\section{Conclusions}

The RD39 collaboration has been developing Charge Injected Detectors (CID) for the past ten years. Normal strip or pixel devices can be operated in CID mode. The requirements for CID operation are a relatively low temperature, e.g. $-50^{\circ} \mathrm{C}$, and a high concentration of deep trapping levels, which means in practise irradiation fluences more than $5 \times 10^{15} \mathrm{n}_{\mathrm{eq}} / \mathrm{cm}^{2}$. At Super-LHC fluences, the CID detectors show CCE more than two times higher than devices operated with reverse bias. Full size CID detectors have been tested in a $230 \mathrm{GeV} / \mathrm{c}$ muon beam at CERN H2 area by using CMS readout electronics and DAQ. The test beam results show that a CID 
detector irradiated at $5 \times 10^{15} \mathrm{n}_{\mathrm{eq}} / \mathrm{cm}^{2}$ shows CCE more than $30 \%$ and a signal-to-noise ratio slightly below ten at $-53^{\circ} \mathrm{C}[16]$.

Other sensor developments focused on LHC luminosity upgrade include $\mathrm{n}$ on $\mathrm{p}$ devices, three dimensional (3D) sensors and high voltage operation of these devices. Latest results were reviewed at the Vertex 2011 conference [17,18] and additionally by common publications of CERN RD50 Collaboration [for instance 19,20].

\section{References}

[1] RD39 Status Report 2004, CERN-LHCC-2004-034 ; LHCC-RD-006.

[2] V. Eremin, J. Härkönen, P. Luukka, Z. Li, E. Verbitskaya, S. Väyrynen and I. Kassamakov, Nuclear Instruments and Methods in Physics Research A581 (2007) 356-360.

[3] RD39 Status Report 2008, CERN-LHCC-2008-019; LHCC-SR-001

[4] Z. Li et al, Nuclear Instruments and Methods in Physics Research A579 (2007) 775-781.

[5] . Eremin, J. Härkönen, Z. Li and E. Verbitskaya, Nuclear Instruments and Methods in Physics Research A583 (2007) 91-98.

[6] M. French, et al., Nucl. Instr. and Meth. A 466 (2001) 359.

[7] J. Härkönen, V. Eremin, E. Verbitskaya, S. Czellar, P. Pusa, Z. Li and T.O. Niinikoski, NuclearA. Instruments and Methods in Physics Research A583 (2007) 347-350.

[8] V. Eremin, Nuclear Instruments and Methods in Physics Research A372 (1996) 388-398.

[9] V. Eremin et al., Nuclear Instruments and Methods in Physics Research A372 (1996) 188.

[10] B. Dezillie, et al., Nuclear Instruments and Methods in Physics Research A452 (2000) 440.

[11] J. Härkönen et al., Nuclear Instruments and Methods in Physics Research A535 (2004) 384.

[12] The CMS Collaboration (R. Adolphi et al.), "The CMS experiment at the CERN LHC", 361pp. JINST 3:S08004, 2008.

[13] T. Mäenpää et al., Nuclear Instruments and Methods in Physics Research A 593 (2008) 523.

[14] P. Luukka et al., Nuclear Instruments and Methods in Physics ResearchA 612 (2010) 497-500.

[15] T. Mäenpää, M. J. Kortelainen, and T. Lampén , IEEE Transactions on Nuclear Science, vol. 57, issue 4, pp. 2196-2199

[16] J. Härkönen et al., Nuclear Instruments and Methods in Physics ResearchA 658 (2011) 51-54.

[17] A. Affolder, "Latest developments in planar n-on-p sensors", Vertex 2011, 19-24 June 2011 Rust, Austria.

[18] R. Bates, "3D Detectors”, Vertex 2011, 19-24 June 2011 Rust, Austria.

[19] A. Affolder et al, Nuclear Instruments and Methods in Physics ResearchA 658 (2011) 11-16. 
[20] J. Metcalfe and the RD50 Collaboration, Nuclear Physics B - Proceedings Supplements, Volume 215, Issue 1, June 2011, Pages 151-153. 